\title{
Electrocardiography and left ventricular function: looking beyond hypertrophy
}

\author{
Christina Byrne ${ }^{1} \cdot$ Muthiah Vaduganathan ${ }^{2} \cdot$ Manan Pareek ${ }^{2,3}$
}

Received: 2 December 2018 / Revised: 7 December 2018 / Accepted: 8 December 2018 / Published online: 11 January 2019

(c) The Japanese Society of Hypertension 2019

Electrocardiographic left ventricular hypertrophy (ECG $\mathrm{LVH}$ ), traditionally evaluated using the Sokolow-Lyon (SL) voltage combination or the Cornell voltage-duration product (CP), is an independent predictor of cardiovascular morbidity and mortality. The combination of these two ECG approaches as well as the inclusion of the strain pattern may further improve risk stratification [1,2]. Therefore, despite the substantial discordance with echocardiographicallydefined LVH, ECG LVH is considered a prognostically important tool [3]. Indeed, the recently published 2018 European Society of Cardiology / European Society of Hypertension (ESC/ESH) guidelines on arterial hypertension recommend a 12-lead ECG for all patients with hypertension and include LVH defined as an SL voltage combination $>35 \mathrm{~mm}$, or $\mathrm{R}$ in a $\mathrm{VL} \geq 11 \mathrm{~mm}$, or as a $\mathrm{CP}$ $>2440 \mathrm{~mm} \times \mathrm{ms}$, or Cornell voltage $>28 \mathrm{~mm}$ in men or $>20$ $\mathrm{mm}$ in women as a criterion for hypertension-mediated organ damage [2]. Conversely, evaluation of cardiac structure and function using transthoracic echocardiography is only recommended if the obtained information is likely to affect treatment decisions [2]. Moreover, the ability of the ECG to provide information on LV function is not clearly established.

The cross-sectional study by Ishikawa et al. expands our knowledge on the relationships between ECG LVH and echocardiographically-determined LV structural and, in particular, functional measures [4]. In a cohort of 288 consecutive outpatients with hypertension, the investigators

Manan Pareek

mananpareek@dadlnet.dk

1 Department of Cardiology, The Heart Centre, RigshospitaletCopenhagen University Hospital, Copenhagen, Denmark

2 Brigham and Women's Hospital Heart \& Vascular Center, Harvard Medical School, Boston, MA, USA

3 Department of Cardiology, North Zealand Hospital, Hillerød, Denmark found comparable and significant associations between left ventricular mass index (LVMI) and SL, LVMI and CP, and LVMI and Cornell voltage. They further demonstrated significant associations between ECG LVH and echocardiographic LV strain measures. Notably, there were modest, but significant correlations between global longitudinal strain (GLS) and SL $(r=0.13, P=0.03)$ and between GLS and CP $(r=0.28, P<0.001)$, as well as GLS and Cornell voltage $(r=0.25, P<0.001)$. Significant associations were also seen between the inner-to-outer ratio of circumferential strain (CS) and CP and between CS and Cornell voltage, but not between CS and SL. Finally, no significant associations were detected for radial strain (RS). The main findings are summarized in Fig. 1.

The relationships were further evaluated in multivariable linear regression models, using the echocardiographic parameters as the dependent variables and SL, CP, and other important covariates as independent variables. Using this approach, the authors found that GLS was significantly associated with either $\mathrm{CP}$ (standardized beta $0.24, P=$ 0.002 ) or Cornell voltage (standardized beta $0.21, P=$ $0.008)$, but not with SL $(P>0.05)$. Considering CS, only Cornell voltage remained significant.

These ECG-derived measures (SL and the two Cornell variables) appear to provide unique information regarding LV structure and function. They have distinct sensitivities and specificities for echocardiographic $\mathrm{LVH}$, their association with echocardiographic LVH is affected by different constitutional risk factors, and they complement each other with respect to risk prediction $[1,3]$. The authors' use of the modified SL criterion $(>38 \mathrm{~mm})$ and possibly, the female correction factor of $6 \mathrm{~mm}$ instead of $8 \mathrm{~mm}$ for $\mathrm{CP}$, might have influenced the diagnostic accuracy of the ECG, given that these thresholds were derived from the Losartan Intervention For Endpoint (LIFE) Reduction in Hypertension Study, in which baseline blood pressures were substantially higher than in the present study [5]. Furthermore, given the testing of multiple associations, the findings are to be considered hypothesis-generating. 


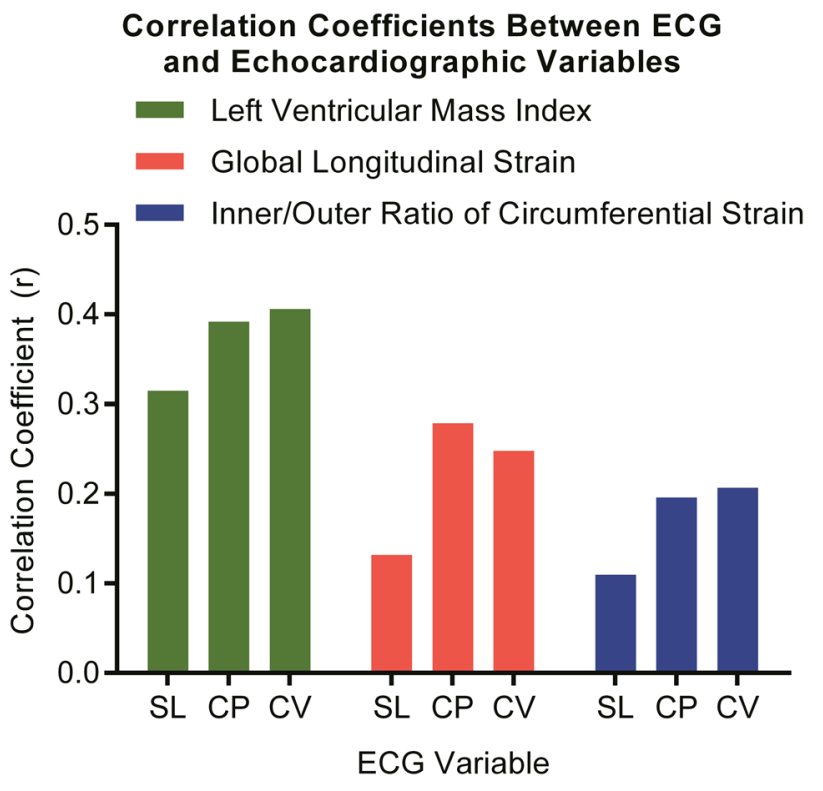

Fig. 1 Correlation coefficients between ECG and echocardiographic variables in the study by Ishikawa et al. [4]. CP Cornell voltageduration product; CV Cornell voltage; SL Sokolow-Lyon voltage combination

Myocardial deformation imaging, particularly when based on speckle tracking, has gained considerable attention over the last decade. Due to the semi-automatic techniques employed, the intra- and inter-observer variabilities in assessing LV function are low when compared with conventional echocardiographic measures like ejection fraction [6]. GLS, typically calculated as the average of the segmental shortening of the myocardium relative to its original length, has important independent and incremental prognostic implications, which have been demonstrated in a variety of settings, including the general population [7].

In the Copenhagen City Heart Study, GLS predicted a composite of heart failure, acute myocardial infarction, and cardiovascular death in men, but not in women [7]. Therefore, a future sex-stratified analysis of ECG LVH and GLS would be desirable. Further considering the fact that sex may modify the correlation between ECG and echocardiographic LVH according to the ECG criteria employed, it is possible that the individual associations between GLS and CP and between GLS and SL differ between men and women [3]. Given the findings from the Malmö Preventive Project Re-Examination Study, these associations could also be affected by age [3]. In another study of elderly Swedish men, LVH assessed by ECG and echocardiography predicted total and cardiovascular mortality independently of each other [8]. The relevant question thus arises whether ECG LVH fully captures the risk signified by GLS and other novel echocardiographic parameters; this could result in a more cost-effective way of approaching patients with otherwise uncomplicated hypertension [2]. However, intuitively, this is unlikely to be the case as the correlations between ECG LVH and strain parameters were at best moderate, and the latter are very sensitive markers of LV function [4].

In summary, the observed associations between ECG markers of LVH and echocardiographic strain parameters are of great interest. In light of the well-known positive impact of ECG LVH regression during antihypertensive treatment on cardiovascular events, future studies should assess how LVH regression is correlated with longitudinal improvements in myocardial strain and other functional parameters $[9,10]$. Studies elucidating the temporal association between ECG LVH with impaired GLS as well as ejection fraction are also needed. Finally, differing criteria for hypertension in Europe and North America may introduce varying prevalence and prognostic estimates of adverse ECG and echocardiographic findings among patients with and without hypertension [11]. Ishikawa et al. advance our understanding of cheap and widely accessible measures to characterize LV function to meet the growing global burden of hypertension.

\section{Compliance with ethical standards}

Conflict of interest MV is supported by the KL2/Catalyst Medical Research Investigator Training award from Harvard Catalyst (NIH/ NCATS Award UL 1TR002541), and serves on advisory boards for AstraZeneca, Bayer AG, and Baxter Healthcare. MP discloses the following relationships_advisory board: AstraZeneca; speaker's fee: AstraZeneca.

Publisher's note: Springer Nature remains neutral with regard to jurisdictional claims in published maps and institutional affiliations.

\section{References}

1. Okin PM, Hille DA, Kjeldsen SE, Devereux RB. Combining ECG criteria for left ventricular hypertrophy improves risk prediction in patients with hypertension. J Am Heart Assoc 2017;6.

2. Williams B, Mancia G, Spiering W, Agabiti Rosei E, Azizi M, Burnier M, et al. 2018 ESC/ESH guidelines for the management of arterial hypertension. Eur Heart J. 2018;39:3021-104.

3. Petersen SS, Pedersen LR, Pareek M, Nielsen ML, Diederichsen SZ, Leosdottir M, et al. Factors associated with diagnostic discrepancy for left ventricular hypertrophy between electrocardiography and echocardiography. Blood Press. 2017;26:54-63.

4. Ishikawa J, Yamanaka Y, Watanabe S, Toba A, Harada K. Cornell product in electrocardiogram is related to reduced LV regional wall motion. Hypertens Res. https://doi.org/10.1038/s41440-0180164-8. e-pub ahead of print 12 December 2018.

5. Dahlof B, Devereux RB, Julius S, Kjeldsen SE, Beevers G, de Faire U, et al. Characteristics of 9194 patients with left ventricular hypertrophy: the LIFE study. Losartan intervention for endpoint reduction in hypertension. Hypertension. 1998;32:989-97. 
6. Farsalinos KE, Daraban AM, Unlu S, Thomas JD, Badano LP, Voigt JU. Head-to-head comparison of global longitudinal strain measurements among nine different vendors: the EACVI/ASE inter-vendor comparison study. J Am Soc Echocardiogr. 2015;28:1171-81, e2.

7. Biering-Sorensen T, Biering-Sorensen SR, Olsen FJ, Sengelov M, Jorgensen PG, Mogelvang R, et al. Global longitudinal strain by echocardiography predicts long-term risk of cardiovascular morbidity and mortality in a low-risk general population: the Copenhagen city heart study. Circ Cardiovasc Imaging. 2017;10. pii: e005521. https://doi.org/10.1161/CIRCIMAGING.116.005521.

8. Sundstrom J, Lind L, Arnlov J, Zethelius B, Andren B, Lithell HO. Echocardiographic and electrocardiographic diagnoses of left ventricular hypertrophy predict mortality independently of each other in a population of elderly men. Circulation. 2001;103:2346-51.

9. Tanabe A, Asayama K, Hanazawa T, Watabe D, Nomura K, Okamura $\mathrm{T}$, et al. Left ventricular hypertrophy by electrocardiogram as a predictor of success in home blood pressure control: HOMED-BP study. Hypertens Res. 2017;40:504-10.

10. Wachtell K, Okin PM, Olsen MH, Dahlof B, Devereux RB, Ibsen $\mathrm{H}$, et al. Regression of electrocardiographic left ventricular hypertrophy during antihypertensive therapy and reduction in sudden cardiac death: the LIFE study. Circulation. 2007;116:700-5.

11. Whelton PK, Carey RM, Aronow WS, Casey DE Jr, Collins KJ, Dennison Himmelfarb C, et al. 2017 ACC/AHA/AAPA/ABC/ ACPM/AGS/APhA/ASH/ASPC/NMA/PCNA Guideline for the Prevention, Detection, Evaluation, and Management of High Blood Pressure in Adults: A Report of the American College of Cardiology/American Heart Association Task Force on Clinical Practice Guidelines. Hypertension. 2018;71:e13-e115. https://doi. org/10.1161/HYP.0000000000000065. Epub 2017 Nov 13. 